Primož Potočnik, Edvard Govekar,

Semi-supervised vibration-based classification and condition monitoring of compressors,

Mechanical Systems and Signal Processing 93 (2017) 51-65.

(Available online 16 February 2017)

(C) 2015. This manuscript version is made available under the CC-BY-NC-ND 4.0 license

http://creativecommons.org/licenses/by-nc-nd/4.0/

Link to published article: https://www.sciencedirect.com/science/article/pii/S088832701730047X

DOI: https://doi.org/10.1016/j.ymssp.2017.01.048

\title{
SEMI-SUPERVISED VIBRATION-BASED CLASSIFICATION AND CONDITION MONITORING OF COMPRESSORS
}

Primož Potočnik ${ }^{1}$

University of Ljubljana, Faculty of Mechanical Engineering, 1000 Ljubljana, Slovenia

Edvard Govekar

University of Ljubljana, Faculty of Mechanical Engineering, 1000 Ljubljana, Slovenia

\begin{abstract}
Semi-supervised vibration-based classification and condition monitoring of the reciprocating compressors installed in refrigeration appliances is proposed in this paper. The method addresses the problem of industrial condition monitoring where prior class definitions are often not available or difficult to obtain from local experts. The proposed method combines feature extraction, principal component analysis, and statistical analysis for the extraction of initial class representatives, and compares the capability of various classification methods, including discriminant analysis (DA), neural networks (NN), support vector machines (SVM), and extreme learning machines (ELM). The use of the method is demonstrated on a case study which was based on industrially acquired vibration measurements of reciprocating compressors during the production of refrigeration appliances. The paper presents a comparative qualitative analysis of the applied classifiers, confirming the good performance of several nonlinear classifiers. If the model parameters are properly selected, then very good classification performance can be obtained from NN trained by Bayesian regularization, SVM and ELM classifiers. The method can be effectively applied for the industrial condition monitoring of compressors.
\end{abstract}

\section{Keywords}

Condition monitoring, Reciprocating compressors, Classification, Semi-supervised, Neural networks, Extreme learning machines.

\section{Introduction}

Condition monitoring (CM) of machines and products is an established and important part of successful modern industrial production. In order to manufacture fault-free products, various non-destructive, time

\footnotetext{
${ }^{1}$ Corresponding author.

E-mail address: primoz.potocnik@fs.uni-lj.si (P.Potočnik)
} 
series analysis based condition monitoring approaches can be applied during the production process. Vibration signal analysis [1,2] continues to be one of the most useful and popular CM methods, as well as other acoustic and acoustic emission based approaches [3,4]. Analysis of vibration signals usually requires and combines feature extraction and classification methods. Many feature extraction methods are known for various types of vibration signals, and the methods include statistical and spectral approaches, wavelets [5,6], psychoacoustic features [7], the Wigner-Vile distribution [8], empirical mode decomposition [9], chaotic vibration [10], and others. Classification methods include various statistical methods, neural and fuzzy logic based methods [11,12], and other modern machine learning methods, such as support vector machines $[13,14]$ and, recently, deep learning approaches [15]. A broad overview of the numerous methods under the general title of natural computing, including neural networks, fuzzy logic, support vector machines, and other methods applicable to mechanical systems research, is provided in [16].

In this paper, a vibration-based condition monitoring and fault detection approach for reciprocating compressors installed in refrigeration appliances is discussed. Various condition monitoring methods for different parts of reciprocating compressors have already been proposed. Yang et al. [17] applied neural networks (self-organising feature map and learning vector quantisation) and support vector machines to classify the conditions of reciprocating compressors based on wavelet features extracted from vibration and noise signals. Their results showed good reliability and flexibility in detecting faulty compressors. Elhaj et al. [18] introduced a method for monitoring the condition of reciprocating compressors and their valves using the crankshaft instantaneous angular speed and a cylinder pressure waveform. An experimental study confirmed the results obtained by using numerical simulation. Potočnik et al. [19] proposed a method for the detection of spring faults during the assembly of reciprocating compressors, which causes incorrect positioning of the compressor body on the supporting springs. The method combines analysis of force measurements performed on the manipulating system and statistical analysis by means of adaptive decision strategy. Qin et al. [20] introduced a scheme for the fault detection of compressor valves based on denoising via basis pursuit, feature extraction via wave matching, and classification via a support vector machine. The core idea of these authors was to determine the underlying model of the vibration signal and to choose a suitable parameterized waveform to match the signal. The method can differentiate compressor valve faults with high accuracy and reliability. Lin et al. [21] developed an automated vibration-based fault detection approach for the classification of reciprocating compressors. They applied a genetic algorithm in order to optimize the process, based on features extracted by time-frequency analysis, and a probabilistic neural network for classification. Wang et al. [22] described a method of diagnosing faults in reciprocating compressor valves using the acoustic emission signal coupled with the simulated valve motion, and considered that the proposed method could be used to easily distinguish between various valve conditions. Pichler et al. [23] proposed a novel approach for detecting cracked or broken reciprocating compressor valves under varying load conditions. The approach is based on a spectrogram representation of the vibration measurement data which shows typical patterns, depending on the fault state.

The existing approaches described in the literature are mostly based on supervised learning, where appropriate sets of samples representing classes of normal and faulty operation are available. Such a situation is difficult to find in industrial environments where prior class definitions are often not available. Labelling the condition monitoring data for supervised fault diagnosis and prognosis is expensive due to the use of field knowledge, whereas unlabelled data contain significant information about normal conditions or faults, which cannot be explored by supervised learning [24]. Consequently, statistical approaches, unsupervised learning, or various semi-supervised approaches, can be applied [25-27].

In this paper, novel a semi-supervised approach for condition monitoring and fault detection of reciprocating compressors is proposed, in which statistical evaluation of features is only applied to extract the initial class members, followed by the application of more advanced classification approaches in order to define class boundaries. The paper provides a comparative analysis of the various state-of-the-art 
classification approaches, including discriminant analysis (DA), neural networks (NN), and support vector machines (SVM), and also includes a novel technique, i.e. extreme learning machines (ELM). Various practical problems encountered in the application of advanced non-linear classifiers are addressed, and the paper also challenges the common logic of comparing various advanced classifiers in order to find the best one. The viewpoint expressed in this paper is that many modern machine learning classifiers offer more than enough expressive power to model almost any hard classification problem, so that a more appropriate question would be whether or not these classifiers are applied properly. The comparative qualitative results summarized in this paper illustrate this dilemma, and summarize suggestions on the best way of applying the described methods.

The main contributions of this paper are the following: (1) a novel semi-supervised approach specifically designed for industrial condition monitoring of reciprocating compressors is proposed, (2) the solution approach introduces a unique combination of various feature selection methods, statistical extraction of initial class representatives, principal component analysis, and the application of various advanced classifiers, and (3) extensive qualitative comparative analysis of various state-of-the-art classification approaches (including extreme learning machines) that are analysed with respect to efficient industrial condition monitoring application. The method is highly relevant for industrial application, and is demonstrated on a case study comprising 20,000 vibration measurements of compressors acquired during the industrial production of refrigeration appliances.

The paper is organized as follows: a description of the proposed solution approach is presented in the next section. Section 3 then introduces the vibration-based condition monitoring system that was applied for data acquisition of the vibration measurements. Extraction of the statistical and spectral features is described in section 4, and the application of features to define the initial classes of compressors is explained in section 5. The various classification methods applied in our study are described in section 6 , whereas section 7 presents the classification procedure and summarizes the classification results. Discussion and conclusions are presented in sections 8 and 9, respectively.

\section{Solution approach}

This study considers the problem of industrial vibration-based condition monitoring where large quantities of vibration measurements are available but labelled class information associated with each measurement is not provided. This situation is relatively common in industrial environments where prior class definitions are not available and are difficult to obtain from local experts. Possible product defects, failures or abnormal operation are not known in advance, so that a machine learning approach is applied whose aim is to analyse the data and extract cases of abnormal operation. Such cases, when found, are then analysed by industrial experts and this forms a feedback loop confirming the proposed condition monitoring strategy. In our approach, which was based on vibration measurements, the objective was on the one hand to detect compressors with too high emitted noise levels (which is unpleasant for end customers), and on the other hand to detect compressors with unusual behaviour (possible defects). In the proposed strategy, the lack of structural knowledge about possible defects is compensated for by the very large size of the acquired data base, and by using a data-driven machine learning approach. In our solution approach, we followed the steps described below:

1) Data acquisition, including the design and installation of an industrial data acquisition system for online measurements of the vibrations of reciprocating compressors installed in refrigeration appliances.

2) Feature extraction, based on statistical and spectral methods, providing initial information about the measured compressors. 
3) Principal component analysis of the extracted features in order to reduce the dimensionality of the data.

4) Extraction of initial categories based on the extracted features and representation of data in the space of the selected principal components.

5) Application of classification methods, including linear methods and a broad selection of nonlinear approaches, in order to estimate the class boundaries between normal compressors and compressors with various types of faults.

6) Qualitative assessment of results, based on graphical analysis of the classification results, providing insights into the operation of the applied classification methods, and providing solutions for the implementation of decision strategy in a real industrial environment.

\section{Data acquisition}

A vibration-based condition monitoring system was developed for the automatic online inspection of reciprocating compressors installed in refrigeration appliances. The complete system with the main rack and control monitor, and the electronic and pneumatic components, is shown in Figure 1 . Since 2015, over 20,000 vibration measurements were acquired during regular industrial production. The system is designed to perform the following operations:

- precise positioning of refrigeration appliances,

- positioning of the sensor head on compressors,

- measuring the vibration signals of compressors,

- $\quad$ analysing and storage of the measurement data.

Since various compressor types are available on the production line, a special custom-made flexible sensor head was developed for automated adjustment of the accelerometer to various compressor types. The sensor head, which is shown in Figure 2, is equipped with a highly sensitive accelerometer: PCB 352B. For each compressor, the vibrations are measured over a duration of 2 seconds with a sampling frequency of $25.6 \mathrm{kHz}$. During the measurements, the compressors are considered to operate in a steady-state regime at a constant speed, after a warming-up time of about 10 minutes. All compressors operate in similar operating conditions and are positioned in refrigerating appliances on rubber-based mounting platforms, which provide good mechanical isolation of the compressors and prevent interference with vibration measurements. These factors were therefore not considered to have any significant role in the characterization of compressor faults. Analysis of different types of compressors showed that some minor differences in vibration levels exist, but preliminary experiments confirmed that vibration levels indicating defects, or emitting too much noise according to industrial standards, are considerably higher than the levels emitted by any types of regular compressors.

Figure 2 shows the flexible sensor head (left) and its position on the compressor during the vibration measurements (right). All the measurements were made without any labelled information about the quality of the measured compressor. For this reason this information has to be extracted based on the results of the analysis of the measurements. The resulting database consists of 20,000 unlabelled vibration signals. 


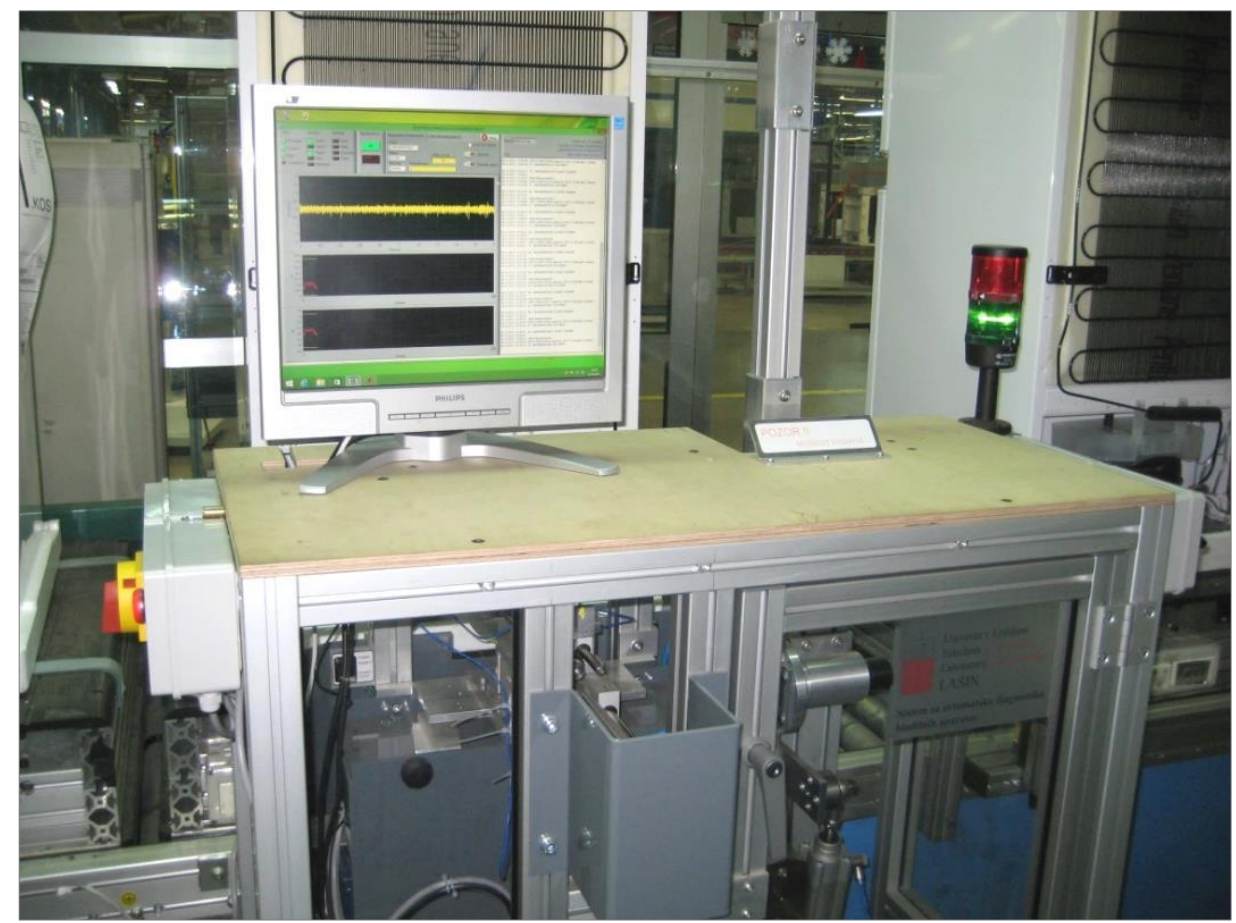

Figure 1: The industrial data acquisition and condition monitoring system.
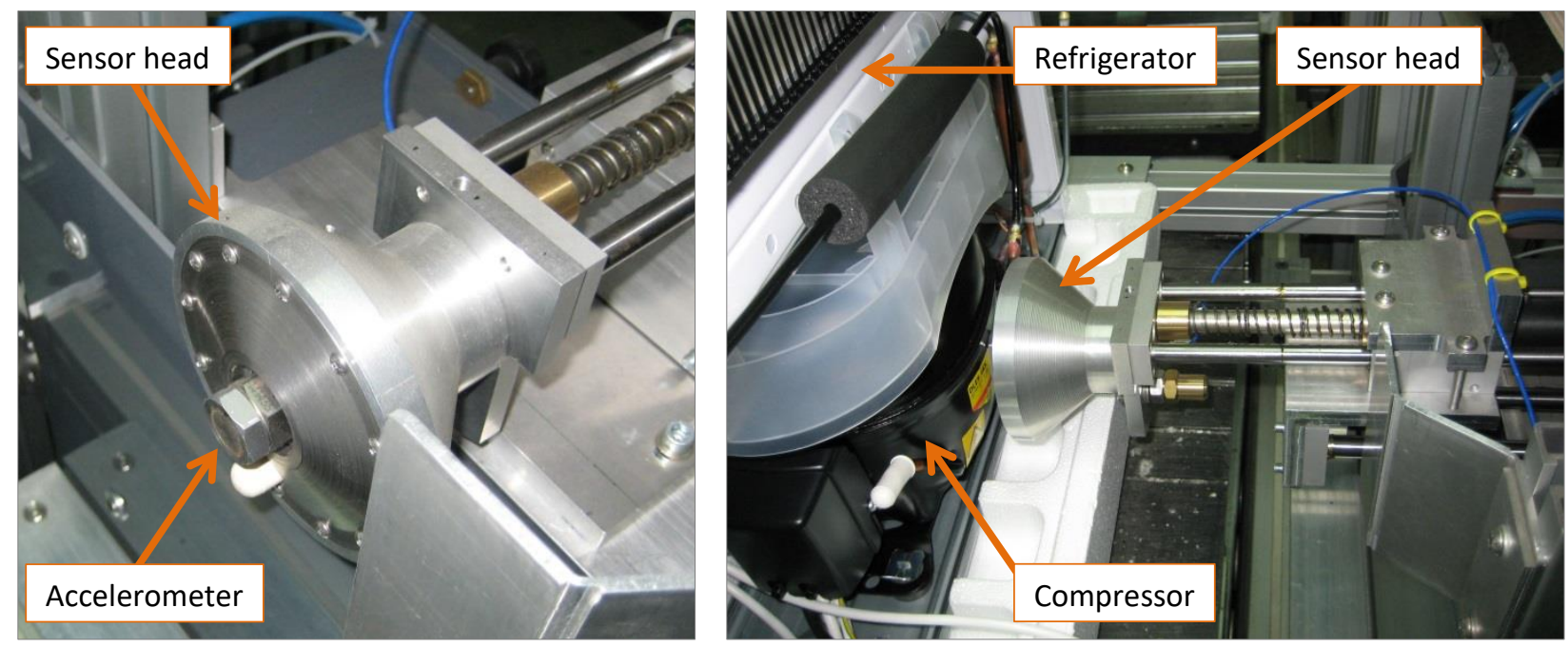

Figure 2: The custom-made flexible sensor head (left) and its position on the compressor during measurements (right).

\section{Feature extraction}

In order to define features for subsequent classification task, two-stage feature extraction was applied. The first stage consisted of the extraction of statistical and spectral features, whereas in the second stage principal component analysis was applied in order to compact the features and further reduce dimensionality. The results of the feature extraction thus provide a transition from the time domain vibration signals into a set of features which characterize each measurement.

\subsection{Extraction of statistical and spectral features}

Various statistical features [28-30], as well as features based on spectral analysis [31], were extracted from the vibration signals in order to provide informative descriptors for the characterization and classification 
task. The exact optimal features are not known in advance, so that our approach combines different features and applies data compression (principal components analysis) and classification methods to solve the task. For each measurement, the following statistical and spectral features were extracted from the vibration signals in the first feature extraction stage:

a) 10 statistical features:

$z_{1}: \quad \log M 1$ (logarithmic mean absolute value of the signal)

$z_{2}, z_{3}, z_{4}: \quad \log M 2, \log M 4, \log M 6$ (logarithmic moments $M_{i}$ ),

$z_{5}: \quad$ kurtosis,

$z_{6}$ : $\quad \operatorname{var} M 2$ (logarithmic variance of M2, calculated across 100 ms segments),

$z_{7}, z_{8}, z_{9}, z_{10}$ : perc 75 , perc 90 , perc 95 , perc 98 (percentiles of absolute values of the signal).

b) 17 spectral features (components $\mathrm{P}_{\mathrm{i}}, \mathrm{i}=1, \ldots, 17$, of power spectral density estimated by Welsh's method):

$z_{11}, \ldots, z_{27}: \quad$ P1 $(0 \mathrm{~Hz})$, P2 $(800 \mathrm{~Hz})$, P3 $(1600 \mathrm{~Hz}), \ldots$, P17 $(12800 \mathrm{~Hz})$.

Most of the features are expressed in logarithmic form, which normalizes the distribution of features. The mean value of the vibration signals is approximately zero, therefore the first feature $\left(z_{1}\right)$ is calculated on the basis of the absolute values of the vibration signals in order to capture useful information. High vibration levels in compressors correspond to high emitted noise levels (that should be detected by the condition monitoring system), so that the feature $z_{1}$ was chosen as an initial class indicator (see also Section 5). This is because it represents a basic indicator of the vibration level, which is also indicative for the acoustic noise levels. Spectral features were calculated as spectral density estimations in equidistant frequency bands, centred at $\{0 \mathrm{~Hz}, 800 \mathrm{~Hz}, 1600 \mathrm{~Hz}, \ldots, 12800 \mathrm{~Hz}\}$. The parameters of these spectral density estimations were chosen as a compromise in order to extract spectral information and to reduce the number of spectral components.

The set of 27 extracted features $\left(z_{1}, \ldots, z_{27}\right)$ for each compressor was further compacted in the second feature extraction stage by principal component analysis.

\subsection{Principal component analysis}

Principal component analysis was applied in order to reduce the dimensionality of a data set consisting of extracted features, while retaining as much as possible of the variation present in the data set [32]. This was achieved by transforming the initial variables into a new set of variables, the principal components (PCs), which are uncorrelated, and are ordered in such a way that the first few components retain most of the variation of the original variables. At this stage, the extracted features were first rescaled to a zero mean and a variance of one, and then the set of 27 standardised features was transformed into 27 principal components. Over $95 \%$ of variability was retained only in the first three principal components, so that only these components were retained for subsequent analyses. Figure 3 shows the composition of the first three principal components which contain $95 \%$ of the variability. 

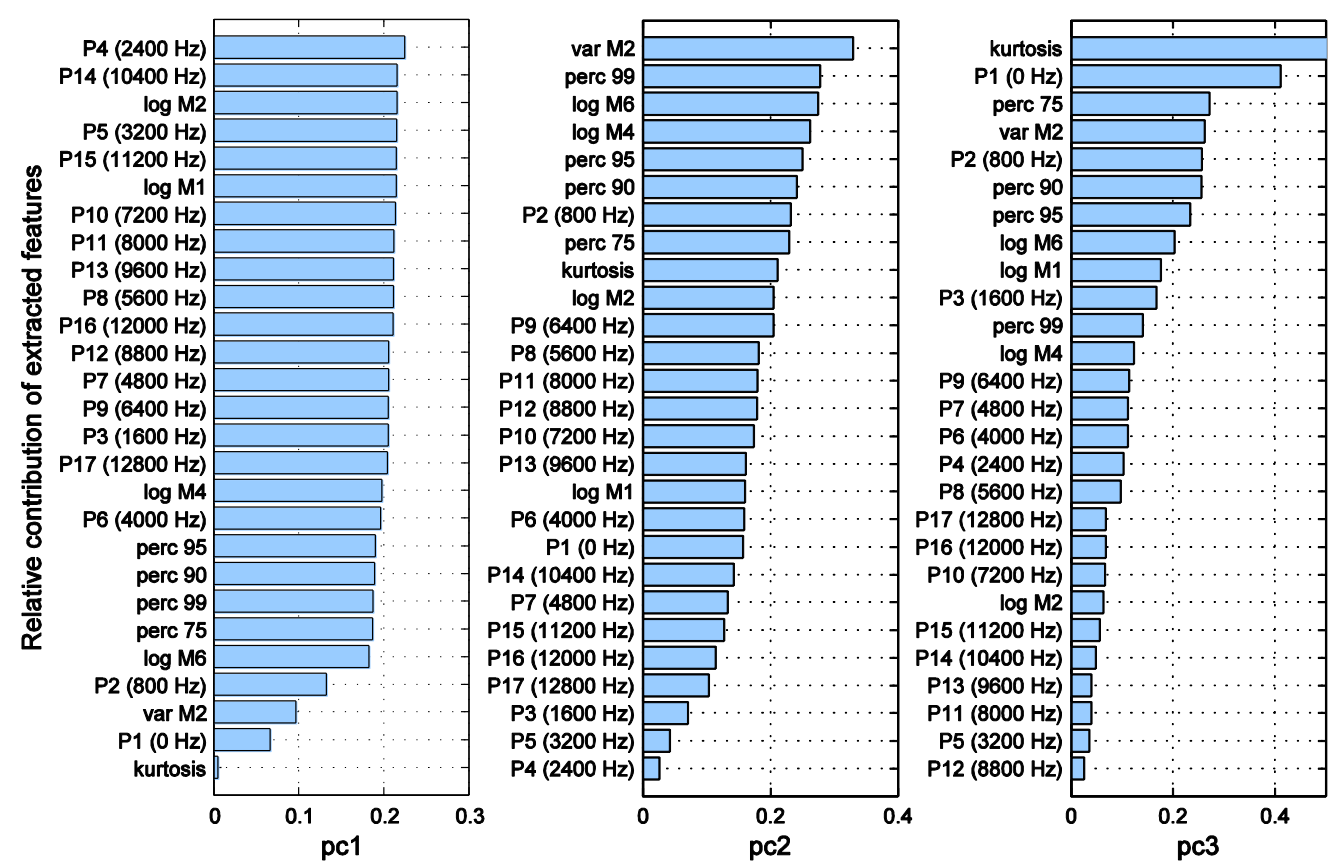

Figure 3: Composition of the first three principal components, derived from the extracted features.

\section{Extraction of initial class information}

The available database contained only unlabelled vibration signals which were acquired online during the production of refrigeration appliances. The classes of compressors of different qualities therefore have to be inferred from the data. In the proposed approach, the feature $z_{1}$ is applied in order to extract initial class information. The selection of this particular feature is not critical, since it is only applied in order to extract initial representatives of classes, and it is not used as a final condition monitoring solution. The feature $z_{1}$ is a basic indicator of the vibration level, and is therefore relevant for condition monitoring. We applied the semi-supervised approach to the classification, as follows:

a) Based on the single selected feature $z_{1}$, the following three initial classes of compressors were statistically defined:

Class 1: normal compressors,

Class 2: noisy compressors,

Class 3: non-operating compressors.

The initial classes $(1,2,3)$ were defined statistically by accepting the $z_{1}$ median 200 samples as normal (class 1 ), a few outliers at the upper $z_{1}$ range as noisy (class 2 ), and 100 samples with the minimum $z_{1}$ values as non-operating (class 3 ). Figure 4 shows the values of the feature $z_{1}$ for the 20,000 samples with the marked initial class $(1,2,3)$ members. 


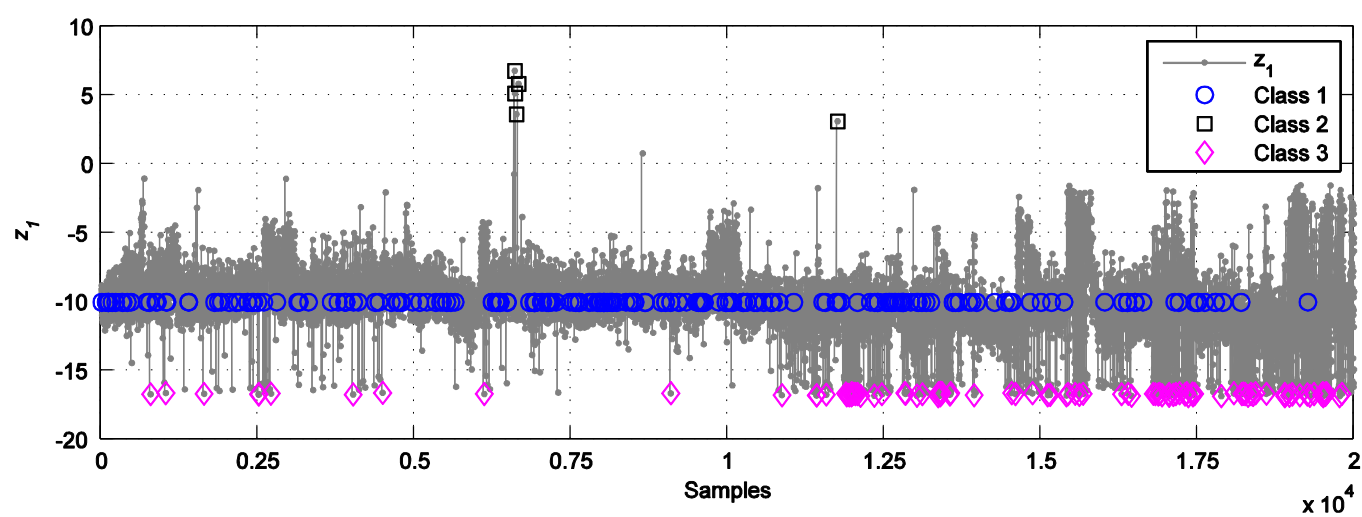

Figure 4: The extracted feature $z_{1}$ for 20,000 samples, with marked initial class $(1,2,3)$ members.

b) As next, the initial classes were plotted in the space of the principal components (Figure 5), which revealed clusters of the defined classes $(1,2,3)$, and also an additional novel class 4 cluster, denoting transient unstable operation:

Class 4: unstable compressors.

For the presentation in PCA projection, the components pc1 and pc3 were selected due to good resolution of the clusters. In Figure 5, all the available samples are plotted as grey dots, whereas the labelled representatives of the initial three classes are marked by blue circles (class 1 ), black boxes (class 2), and magenta diamonds (class 3). After the inspection of the samples in the PCA space, the evidence of the fourth 'unstable' class was confirmed by additional checking of the vibration signals of several samples. These samples were denoted as initial class 4 representatives and marked by red circles in Figure 5.

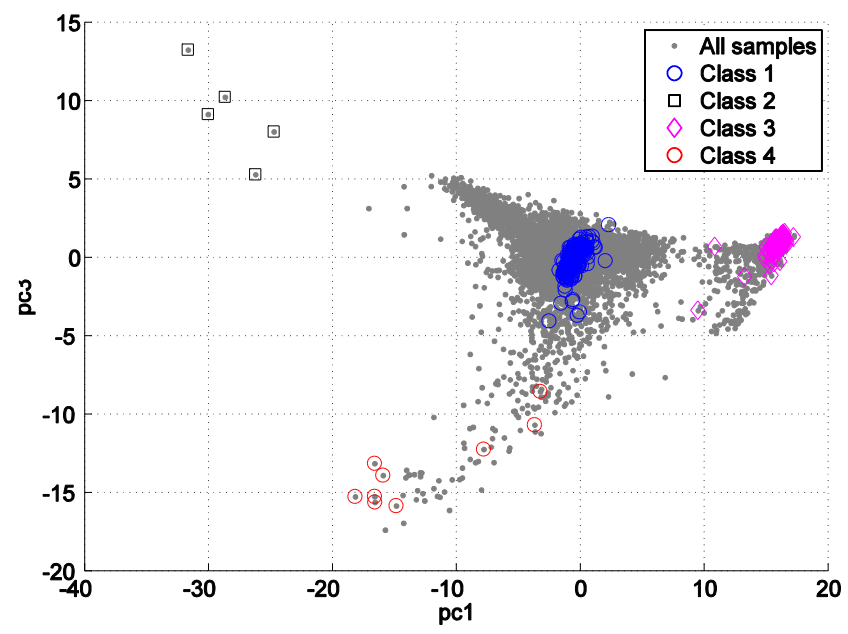

Figure 5: The investigated samples in the space of the $1^{\text {st }}$ and $3^{\text {rd }}$ principal components with the initial class members marked (including the additional class 4).

Figure 6 shows characteristic examples of the vibration signals for all four classes. The accelerometer signals are plotted in relative arbitrary units [a.u.] due to the manufacturer's confidential policy. 

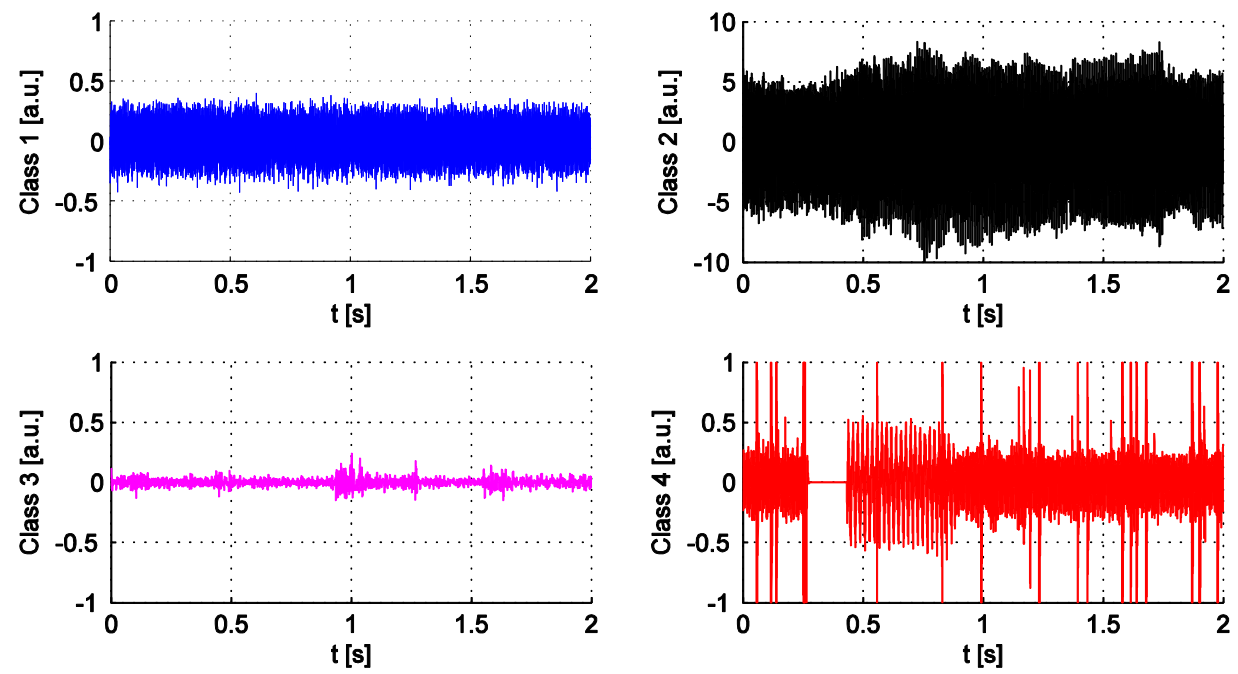

Figure 6: Examples of the vibration signals for all four defined classes of compressors: (1) normal, (2) noisy,

(3) non-operating, and (4) unstable.

\section{Classification methods}

In this section, various machine learning methods for classification are introduced, each with a brief description. The methods include linear discriminant analysis and several contemporary nonlinear classification approaches, such as neural networks, support vector machines, and extreme learning machines. The results by applying these methods are described in the next section, together with a summary of their practical aspects, as well as their benefits and encountered difficulties.

\subsection{Linear discriminant analysis}

Discriminant analysis (DA) is a classification method which is used to determine the discrimination boundaries which separate (i.e. discriminate between) two or more classes of objects [33,34]. DA assumes that different classes generate data based on different Gaussian distributions. DA has been shown to rank among the top classifiers, which is most probably due to the bias-variance trade-off where the data can only support simple decision boundaries such as linear or quadratic, and estimates obtained by Gaussian models are stable [35]. In the case of our analysis, linear discriminant analysis (LDA) was applied. This method is very simple to apply, and does not require the tuning of special method parameters.

\subsection{Neural networks}

The field of neural networks (NN) encompasses a very wide range of various nonlinear modelling structures [36-39]. In our study, several NN models were examined, i.e. feed-forward neural networks (FFNN) with sigmoidal activation functions, and radial basis function networks (RBFN). All the NN models were defined as single hidden layer networks. The number of neurons $L$ in a hidden layer usually represents an open network design parameter.

The output of a FFNN, denoted as $y$, can be represented by a generic expression of the inputs $u$, e.g. in the case of a network with a $K$-dimensional input $\boldsymbol{u}=\left\{u_{1}, u_{2}, \ldots, u_{K}\right\}, L$ neurons in a single hidden layer, and a single output $y$, the FFNN model can be represented as follows:

$$
y=f_{\mathrm{o}}\left(\sum_{j=1}^{L} w_{j} f_{h}\left(\sum_{i=1}^{K} w_{j i} u_{i}\right)\right)
$$

where $f_{o}$ and $f_{h}$ represent the output and hidden layer activation functions, and $w_{j}$ and $w_{j i}$ the output and hidden layer weights. In our study, we applied FFNN with sigmoid output and hidden layer activation functions, and two training algorithms, i.e. the Levenberg-Marquardt algorithm [40] and training with 
Bayesian regularization [41]. The former method is a fast gradient-based method for the very efficient training of neural networks, whereas the latter approach includes regularization in order to prevent overfitting during the learning process.

As an alternative NN solution, we examined also a RBFN model with $L$ hidden layer nodes, which can be described as follows:

$$
y=\sum_{j=1}^{L} w_{j} g\left(\boldsymbol{u}, \boldsymbol{q}_{j}, \sigma\right)
$$

where $g$ represents the radial basis activation function (usually Gaussian), $\boldsymbol{q}_{j}$ the centre of the j-th basis function, and $\sigma$ the width of the Gaussian activation function. For the RBFN training, a standard two-stage training algorithm was applied, which consisted of a selection of radial basis centres, followed by calculation of the output layer weights [42].

The practical application of neural network models requires the prior selection of network architecture (the number of hidden nodes $L$, and in the case of RBFN also the Gaussian width $\sigma$ ). This is usually chosen arbitrarily or based on the results of preliminary cross-validation based experiments. The network training is also considerably affected by random initialization of the network weights, so that the training may converge to various sub-optimal solutions, and several iterations may be needed in order to find good solutions. Consequently, the application of NN requires a certain level of expertise so that the NN solutions are properly designed and trained.

\subsection{Support vector machines}

Support Vector Machines (SVM) are based on statistical learning theory and make use of similar model structures to those of NN but with a radically different training approach which always finds a global solution, in contrast to neural networks, where numerous local minima usually exist [43]. SVM theory is well established, and has been well described in several excellent works [44-46]. The SVM model can be represented by a linear combination of $L$ kernel functions denoted by $\Phi_{j}$, weights $w_{j}$, and a bias $b$ :

$$
y=\sum_{j=1}^{L} w_{j} \phi_{j}(\boldsymbol{u})+b
$$

In this study, SVM with linear kernel functions and radial basis kernel functions were applied. The calculation of a SVM solution depends on a generalization parameter $C$ and a kernel parameter $\gamma$. The optimal values of both parameters are arbitrary and should be determined numerically by a cross-validation method over a wide range of possible values. This complicates the practical application of SVM, since there is not much orientation about proper values of the parameters $(C, v)$. However, once these parameters have been fixed, the SVM converges to a global (optimal) solution. It should, however, also be noted that the radial basis function network, once its centres $\boldsymbol{q}$ and widths are $\sigma$ fixed, always converges to a global solution.

\subsection{Extreme learning machines}

Extreme learning machines (ELM) have been recently proposed as a new fast learning algorithm for single layer feed-forward networks (SLFN) [47]. The ELM algorithm randomly chooses hidden layer nodes, and analytically determines the output weights of the SLFN. This algorithm tends to provide good generalization performance at an extremely fast learning speed. A broad overview of various ELM applications has been summarized in [48], and an extensive review of a current state of ELM has been presented in [49]. The ELM model can be defined by the following expression:

$$
y=\sum_{j=1}^{L} w_{j} h(\boldsymbol{u})
$$

where $w_{j}$ represents the output weights, and $h$ the nonlinear feature mapping, which is defined as a nonlinear piecewise continuous function which satisfies the ELM universal approximation theorems [50, 51]. 
Commonly used ELM mapping functions include sigmoid, hyperbolic tangent, Gaussian, multiquadratic, and cosine functions. ELM train an SLFN in two main stages:

(1) random feature mapping, and

(2) solving linear parameters.

In the first stage, ELM randomly initializes the hidden layer to map the input data into an ELM feature space by means of the nonlinear mapping functions $(h)$. In the second stage of ELM learning, the weights $w_{j}$ connecting the hidden layer and the output layer are solved by minimizing the approximation error in the squared error sense which leads to a global solution. A comparative analysis of SVM and ELM [52] has shown similar generalization abilities of both methods for larger training sets.

\section{Classification results}

This section presents the classification results obtained by applying the described classification methods to extracted initial classes of compressors (see Section 5). The extracted principal components pc1 and pc3 are used as inputs for the classifiers. The principal components pc1 and pc2 could also be used, but the components pc1 and pc3 offer slightly better graphical representation of the separation of classes. The proposed methodology is general, and can be applied to more than two inputs. The application of more than two inputs would only improve the separation of classes, but this was not required for our study, which was limited to two inputs ( $\mathrm{pc} 1$ and $\mathrm{pc} 3$ ) since they already offer good separation of classes, and two-dimensional inputs also enable good graphical representation which is important in order to gain an understanding of the operation of discussed classifiers.

For all the classifiers, the procedure is performed as follows:

a) Labelled samples with initially defined four classes are used as training data for the classifiers. In our case study, the training data amounts to 314 samples, containing 200 samples of class 1 (normal compressors), 5 samples of class 2 (noisy compressors), 100 samples of class 3 (non-operating compressors), and 9 samples of class 4 (unstable compressors). The training data set thus contains four class subsets with a very small number of class representatives with different probabilities of occurrence. For this reason the classification task is difficult but very relevant for industrial condition monitoring applications.

b) Based on the labelled training data, the classifiers are constructed and trained. Due to the very limited training data set (only 314 labelled samples compared to 20,000 available vibration measurements), the emphasis is on the robustness and generalization ability of the classifiers.

c) Finally, the trained classifiers are applied across a wider input range (which includes all the available samples). The classification results are graphically represented with colours that denote different regions according to particular class membership.

d) Evaluation of the classification results: all the presented classifiers solve the training problem correctly (except in cases which deliberately show unsuccessful performance). Due to the semisupervised approach with missing class labels for the majority of the available data, it is impossible to quantitatively evaluate the classifier's performance. But with the available graphical representation, it is possible to qualitatively estimate the expressive power, classification ability, and generalization performance of each classifier. This is a rather unusual approach, but the authors believe that it is very relevant for industrial applications, and also very informative in the sense that it provides valuable information about the characteristics and performance of each considered classifier. 
The presentation of the classification results begins with a linear discriminant analysis, which is shown in Figure 7, presenting the samples in the space of the principal components $p c 1$ and pc3 together with the decision boundaries. All the available samples are plotted as grey dots, whereas the labelled representatives of each class are marked by blue circles (class 1 ), black boxes (class 2 ), magenta diamonds (class 3 ), and red circles (class 4). The decision boundaries between each faulty class (classes $2,3,4$ ) and the normal class (class 1) obtained by LDA are plotted as black lines. The LDA partitions the feature space into linear segments that already sufficiently solve the classification task.

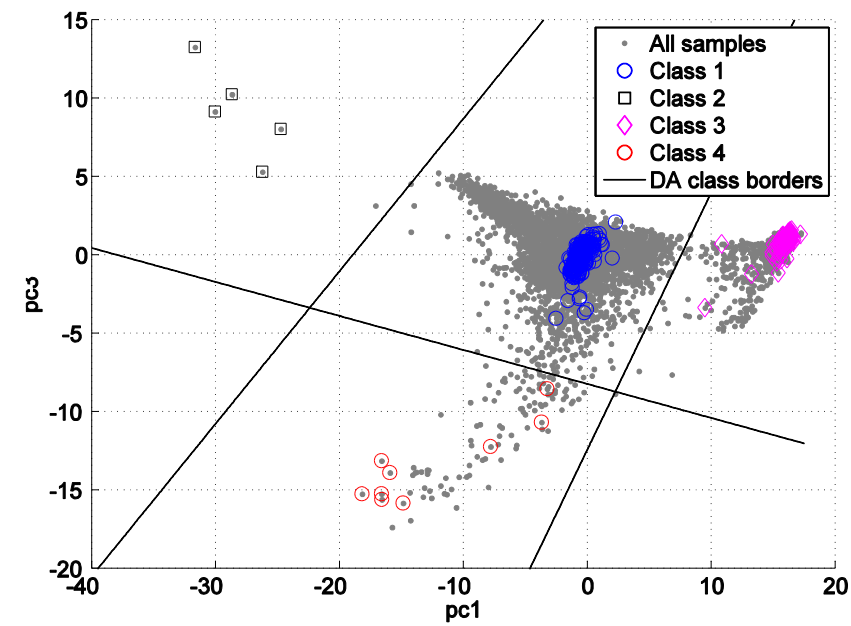

Figure 7: Classification by means of linear discriminant analysis.

Next, Figures 8-10 present various classification results obtained by the neural network models. Figure 8 shows two solutions obtained by a feed-forward neural network with 8 sigmoidal hidden layer nodes, trained by Levenberg-Marquardt back-propagation. Many repeated iterations of learning, started by random initialization and followed by Levenberg-Marquardt back-propagation, revealed that the learning process often stalled in local non-optimal solutions, such as that shown in Figure 8 left, and only occasionally converged to a meaningful global solution, such as shown in Figure 8 right. The Levenberg-Marquardt algorithm is very fast and efficient, and can be considered as a contemporary standard training method for neural networks. However, it is not a suitable method for our case study due to the very small labelled training set. It should be noted that false learning convergence (such as Figure 8 left) can easily be detected either visually (in a case of two-dimensional inputs) or numerically by a very low correct classification rate.
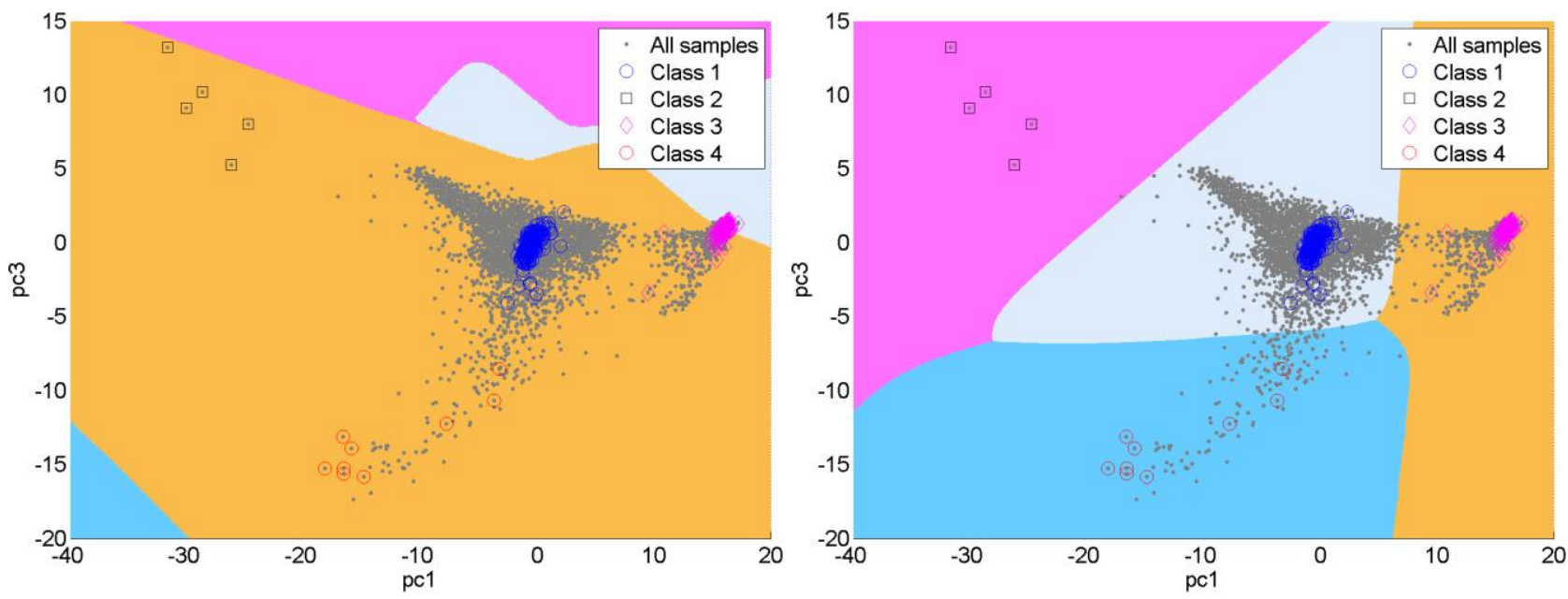

Figure 8: Classification with a feed-forward neural network with 8 hidden neurons, trained by LevenbergMarquardt back-propagation, does not always converge to a meaningful solution. 
Considerable improvements in robustness can be achieved by introducing Bayesian regularization into a Levenberg-Marquardt algorithm. In this case the training converges very consistently to robust optimal classification solutions, as shown in Figure 9 (left and right). Both solutions were obtained by the same NN structure (8 sigmoidal hidden layer neurons) as in the case of Figure 8, but trained by added regularization. For a small data set such as was provided in our case study, feed-forward neural network trained by regularization is a very good solution which consistently provides meaningful near-optimal classification results.
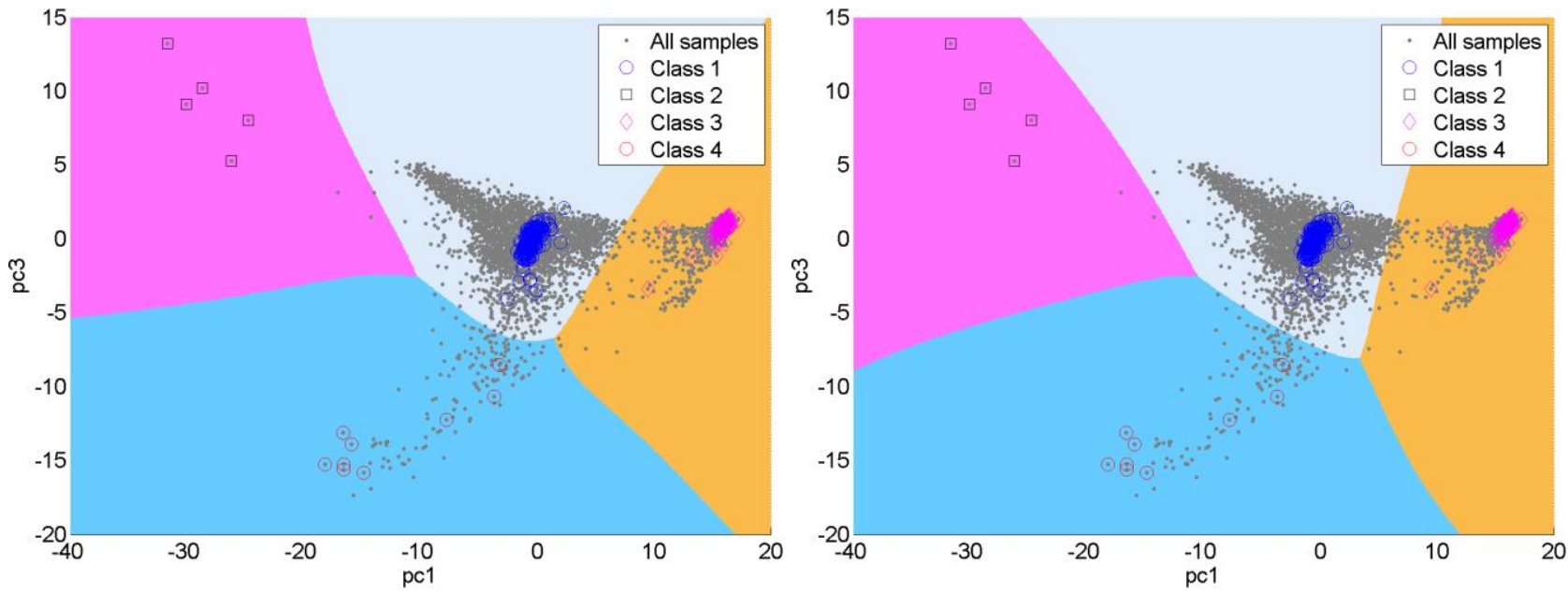

Figure 9: Classification with a feed-forward neural network with 8 hidden neurons, trained by a LevenbergMarquardt algorithm combined with Bayesian regularization, consistently provides good solutions.

As the last NN solution, a structurally different $\mathrm{NN}$ can be applied, i.e. a radial basis function network. In this case the model consists of radial basis function hidden layer neurons. Figure 10 shows the classification results for RBFN with $L=4$ hidden layer neurons (left), and $L=20$ hidden layer neurons (right). Both results were obtained by using a wide spread parameter $\sigma=100$. The RBFN model can provide excellent classification solutions (such as that shown in Figure 10 left), if only the model parameters $(L, \sigma)$ are properly chosen, which is not a trivial task. But the good news is that once the RBFN structure with hidden layer centres and spreads is fixed, the learning always converges towards an optimal solution. Thus, with its two-stage training process, the RBFN structure effectively overcomes the local convergence problem, which is typical for sigmoidal NN trained by back-propagation. 

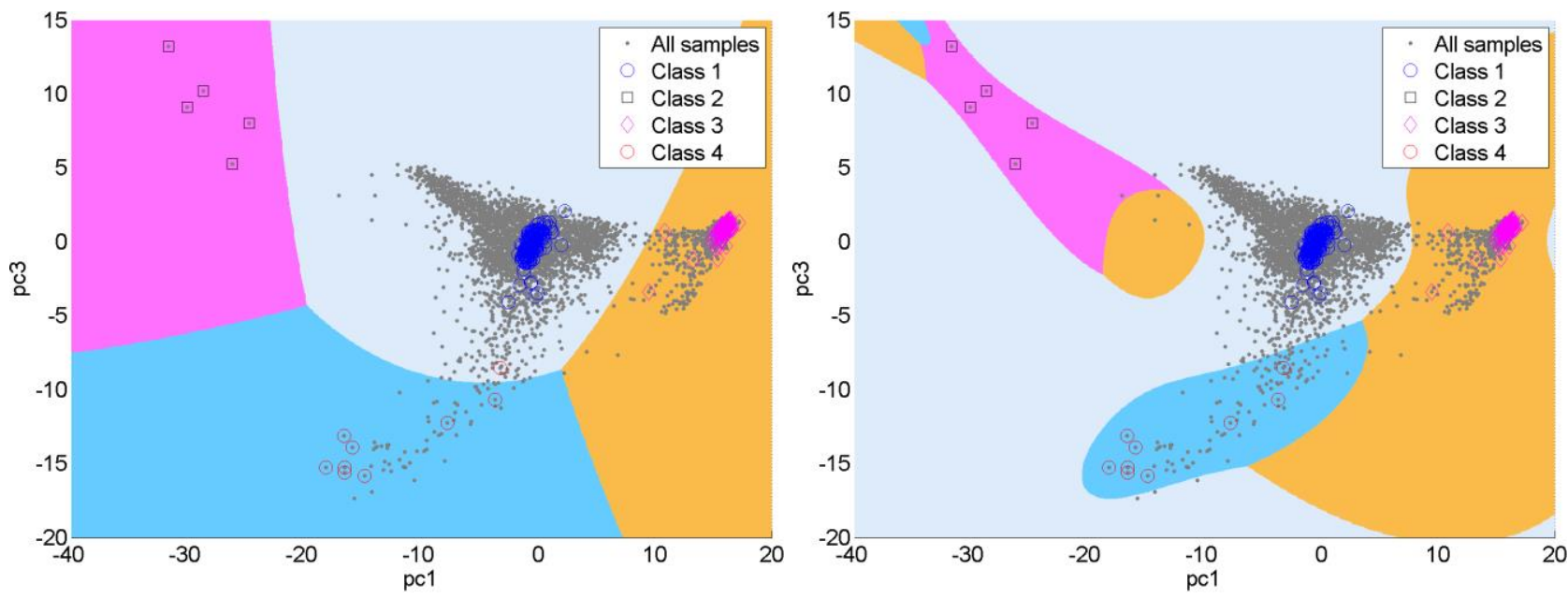

Figure 10: RBFN classification with 4 and 20 hidden units. The results are very sensitive to proper selection of the model parameters (the number of hidden units, and the spread).

The next set of solutions is provided by the SVM models. These models overcome the usual structural challenges typical for NN where selection of the model structure and its parameters considerably influences the final solution. The construction of a SVM model does not require any pre-conditioned structural design, but it does require the selection of SVM parameters $(C, \gamma)$, which can be defined arbitrarily within a wide range of many magnitudes. However, once $C$ and $\gamma$ are fixed, the SVM converges to a global solution. The first SVM example is shown in Figure 11, which represents a solution of a linear SVM which provides consistent and stable results across a wide range of both SVM parameters. The example shown in Figure 11 was obtained at $C=1 \mathrm{e} 4$ and $y=100$. Linear SVMs offer a very appealing and robust classification solution with good generalization abilities. As well as this, apart from the requirement that proper values of $C$ and $v$ are selected, not many other additional skills are needed if the method is to be properly applied.

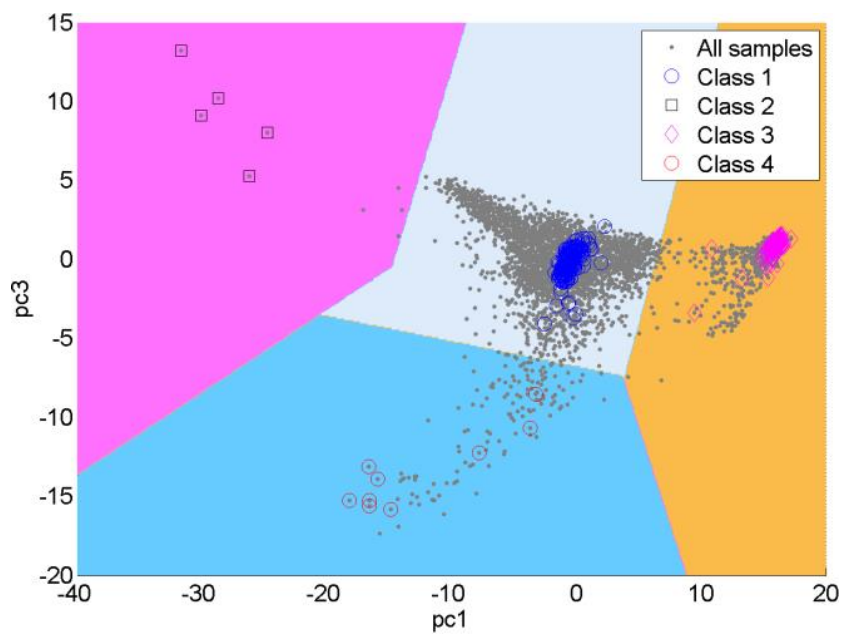

Figure 11: Linear SVM provides consistent and suitable solutions across a wide range of $C$ and $\gamma$ parameters

(the example shown was obtained at $C=1 \mathrm{e} 4$ and $\gamma=100$ ).

More advanced nonlinear classifiers can be constructed by SVMs with nonlinear kernel functions, such as sigmoidal or radial basis functions. An example of a RBF-SVM is shown in Figure 12, where the left plot corresponds to $C=100$ and $y=0.005$, and the right plot to $C=100$ and $\gamma=0.05$. Compared to the linear SVM, the selection of SVM parameters for nonlinear kernels is much more sensitive and requires prior crossvalidation based experiments in order to find the appropriate range. This complicates the application of nonlinear SVM, but this is the price which has to be paid for one of the best contemporary nonlinear 
classifiers. In our case study, where already simple decision boundaries can adequately solve the problem, nonlinear SVMs would not provide additional benefits compared to a linear SVM.
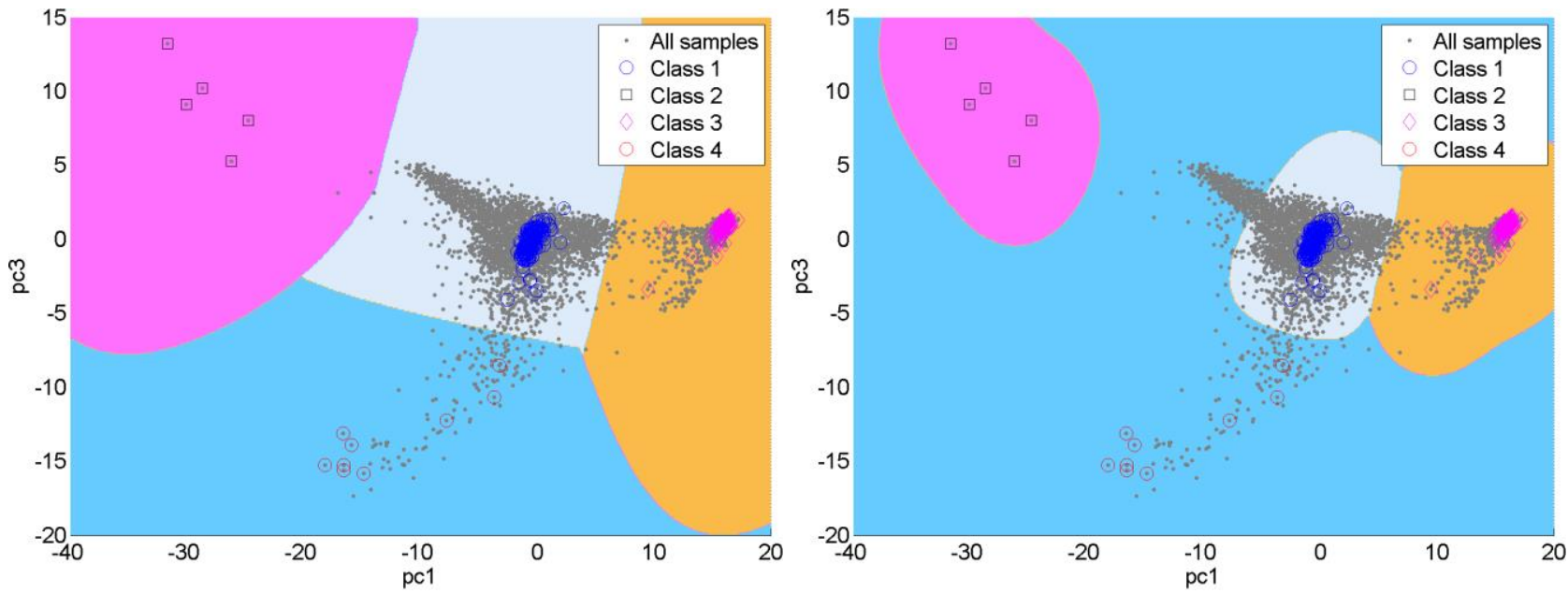

Figure 12: SVMs with RBF kernels are quite sensitive to the selection of appropriate SVM parameters

$(C$ and $\gamma)$.

The last result is shown for the ELM model. The key benefits of ELM models are very fast training due to the randomly chosen hidden layer units, and a good generalization ability. However, the random choosing of hidden layer parameters also has a downside which is that ELM models are quite sensitive to the range of input variables since the hidden layer parameters are not tuneable [53]. For this reason with respect to generalization ability results may vary for different ranges of input variables, since in each case different regions of the ELM nonlinear activation functions are excited. Figure 13 presents the results for an ELM model with 100 sigmoid hidden nodes, where the inputs were rescaled (multiplied) with a scaling factor $x_{\mathrm{s}}=1 \mathrm{e}-4$ (Figure $13 \mathrm{left}$ ) and $x_{\mathrm{s}}=1 \mathrm{e}-6$ (Figure 13 right). The results confirm the very good classification and generalization ability of ELM models, but only in the case of properly rescaled inputs.
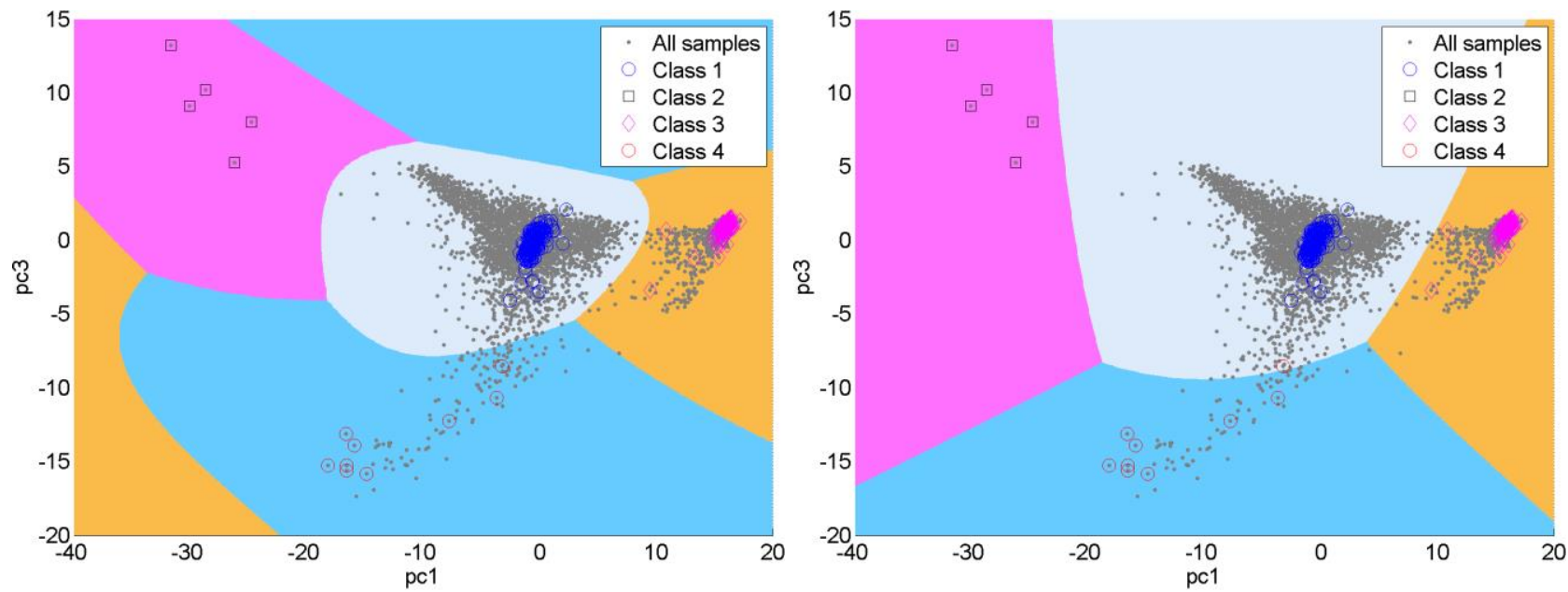

Figure 13: ELM classification with 100 sigmoid hidden nodes provides robust results across a wide range of input scaling (left: $x_{\mathrm{s}}=1 \mathrm{e}-4$, right: $\left.x_{\mathrm{s}}=1 \mathrm{e}-6\right)$.

\section{Discussion}

The comparison of the classification results presented in the previous section provides some interesting insights into the operation of different classification methods, as well as opening up various questions. Figure 7 shows that already the linear DA method solves the task sufficiently well, which raises the question: 
why complicate matters by using more sophisticated nonlinear methods? There seems to be a two-fold answer to this question: firstly, the decision boundaries provided by the LDA method may not be optimal, so that more advanced methods need to be investigated, and secondly, the presented case study cannot be extrapolated to more complex problems which require solutions that are beyond linear separability. For these two reasons more complex nonlinear solutions need to be taken into consideration in order to satisfy generality and to achieve the ability to more optimally solve the task.

A comparison of the results obtained by traditional neural network solutions (Figure 8) shows that these methods offer a generalization ability for solving nonlinear problems, but this by itself does not guarantee optimal solutions due to the trapping of iterated gradient-based learning into local attractors (local minima of the training error). The authors of this study therefore recommend that neural network training is additionally supported by regularization methods in order to ensure smooth solutions with good generalization. As shown in Figure 9, properly designed neural networks trained by Bayesian regularization consistently provide good classification results, and can therefore be recommended for industrial applications. RBFN solutions (Figure 10) do not suffer from local trapping problems, and can provide good classification solutions, but only if the network parameters are properly chosen, which is not straightforward and may require a parameter search based on the cross-validation approach.

In contrast to NN solutions, support vector machines provide fundamentally a different learning framework, which avoids local trapping problems and provides a good approach to the establishment of optimal decision boundaries. This is already evident from the results obtained by linear SVM (Figure 11), which efficiently partitions space into regions corresponding to different compressor qualities, and reliably provides more optimal results than linear discriminant analysis (which is based on a different formulation of optimality). Although more complex than LDA, linear SVM is a powerful classification method, which does not require any special tuning of the model parameters. It earns a high recommendation for industrial applications, but is limited by the linearity of its decision boundaries. In the case of more complex problems, nonlinear SVM can be applied (see Figure 12) but these methods require tuning of the two model parameters $(C, y)$ within an arbitrary wide range, which is usually accomplished by the cross-validation approach. Nonlinear SVMs can provide a powerful classification approach, but they require a certain level of expertise in order to properly tune the solution.

As the last method in our research, we investigated the recently introduced ELM method, which excels with its very fast learning and good generalization properties. ELM operation is comparable to SVM, and consistently provides good classification results, but only if the input range is properly rescaled. Based on the results of our investigation (Figure 13), this scaling is robust across several orders of magnitude and therefore seems not to present much trouble in the design of ELM solutions. As such, the ELM approach also receives our recommendation for industrial practice, particularly because its implementation is simple and does not require the use of sophisticated mathematical toolboxes.

Finally, the way in which the results obtained by the use of nonlinear classifiers is reflected back onto the original extracted feature $z_{1}$ (which was used to extract the initial class information) is presented. In order to do this the classification obtained by the ELM model (Figure $13 \mathrm{left}$ ) is taken, and the obtained classes are marked for all the original samples plotted as $z_{1}$ values. Figure 14 shows all $20,000 z_{1}$ values with their marked class memberships. It can be clearly seen that a single extracted feature cannot provide accurate diagnostic information since many faulty compressors overlap the central region of normal compressors. This figure shows and confirms the benefits of applying more complex classification methods in comparison with a simple single feature-based statistical condition monitoring approach, which is the most often used in industrial applications. 


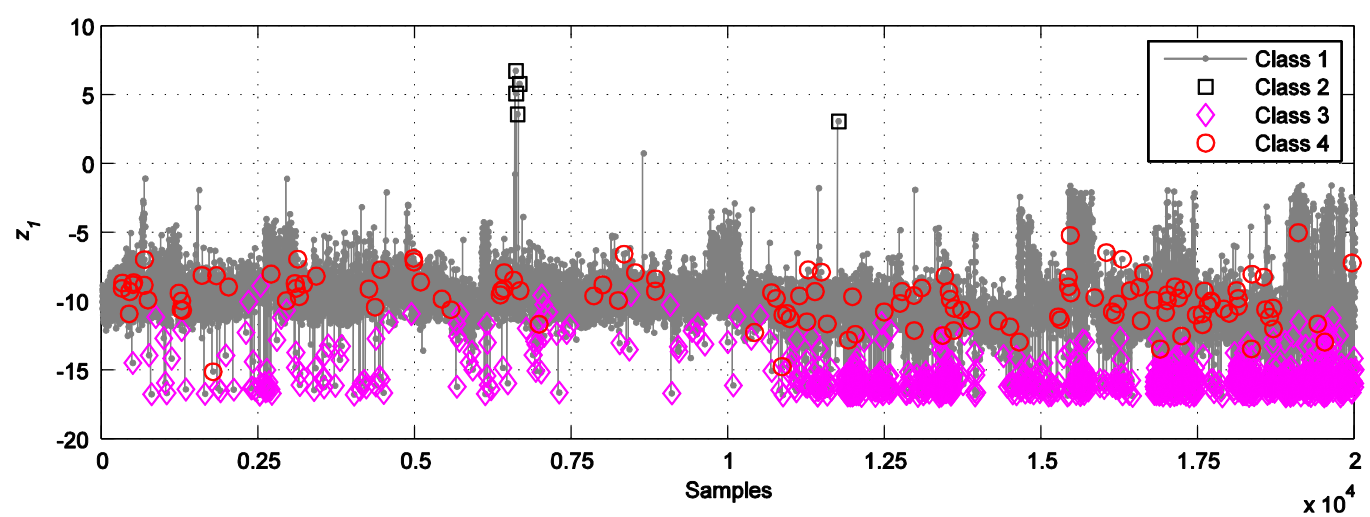

Figure 14: The ELM classification results projected back into the feature $z_{1}$ with the marked classes.

\section{Conclusions}

The paper considers an industrial condition monitoring problem for compressors installed in refrigeration appliances, where a large database of vibration-based measurements is available but without prior class definitions. Therefore, a semi-supervised approach to the vibration-based classification of compressors is proposed. The proposed solution includes feature extraction and principal component analysis as preprocessing stages and tools to extract the initial class information, and then the use of more advanced classification methods in order to define the class boundaries. The paper provides an overview of the many different available classification methods, including various types of neural networks and support vector machines, as well as a relatively new extreme learning machines method. For each classification method, the benefits and difficulties of their practical application are discussed, and the classification results are compared qualitatively based on graphical analysis. The latter provides useful insights into the operation of each classifier.

The paper shows that already linear methods, including linear DA and linear SVM, provide good classification solutions and can be considered as suitable candidates for industrial applications due to their numerical simplicity and stable operation. The paper also confirms the very good performance of several nonlinear classifiers, i.e. neural networks trained by Bayesian regularization, nonlinear SVM, and ELM classifiers. The considered nonlinear classifiers have the ability to cope with much more complex problems, so that they can provide a more general solution approach. However these methods also require the tuning of various model parameters, which is not a trivial task and often requires a certain level of expertise and numerical cross-validation based preliminary simulations. As a relatively new method, extreme learning machines are definitely worth considering as a possible condition monitoring solution approach due to their numerical simplicity, relatively easy application, and good generalization ability. The proposed semisupervised approach can be successfully applied to the industrial condition monitoring of compressors, as is shown in this paper, and also has the potential to solve similar challenges in the field of vibration-based industrial condition monitoring.

\section{Acknowledgements}

This work was supported by ARRS - the Slovenian Research Agency, Research program P2-0241 "Synergetics of complex systems and processes".

\section{References}

[1] R.B. Randall, Vibration-based Condition Monitoring: Industrial, Aerospace and Automotive Applications, John 
Wiley \& Sons, Chichester, 2011.

[2] C. Ruiz-Cárcel, V.H. Jaramillo, D. Mba, J.R. Ottewill, Y. Cao, Combination of process and vibration data for improved condition monitoring of industrial systems working under variable operating conditions, Mech. Syst. Signal Process. 66-67 (2016) 699-714. doi:10.1016/j.ymssp.2015.05.018.

[3] P. Potočnik, E. Govekar, I. Grabec, Acoustic and Acoustic Emission Based Condition Monitoring of Production Processes, in: 2nd World Congr. Eng. Asset Manag. 4th Int. Conf. Cond. Monit., Harrogate, UK, 2007: pp. 16141624.

[4] E. Govekar, J. Gradišek, I. Grabec, Analysis of acoustic emission signals and monitoring of machining processes, Ultrasonics 38 (2000) 598-603.

[5] J. Chen, Z. Li, J. Pan, G. Chen, Y. Zi, J. Yuan, et al., Wavelet transform based on inner product in fault diagnosis of rotating machinery: A review, Mech. Syst. Signal Process. 70-71 (2016) 1-35. doi:10.1016/j.ymssp.2015.08.023.

[6] M.J. Gómez, C. Castejón, Crack detection in rotating shafts based on $3 \times$ energy : Analytical and experimental analyses, Mechanism and Machine Theory 96 (2016) 94-106. doi:10.1016/j.mechmachtheory.2015.09.009.

[7] P. Potočnik, E. Govekar, I. Grabec, P. Mužič, Psychoacoustic approach to machine fault diagnosis, Int. J. Acoust. Vib. 10 (2005) 131-136.

[8] N. Baydar, A. Ball, A comparative study of acoustic and vibration signals in detection of gear failures using WignerVille distributions, Mech. Syst. Signal Process. 15 (2001) 1091-1107. doi:10.1006/mssp.2000.1338

[9] Y. Yang, Y. He, J. Cheng, A gear fault diagnosis using Hilbert spectrum based on MODWPT and a comparison with EMD approach, Measurement 42 (2009) 542-551. doi:10.1016/j.measurement.2008.09.011

[10] A. Soleimani, S.E. Khadem, Early fault detection of rotating machinery through chaotic vibration feature extraction of experimental data sets, Chaos, Solitons and Fractals. 78 (2015) 61-75. doi:10.1016/j.chaos.2015.06.018.

[11] P. Subbaraj, B. Kannapiran, Fault detection and diagnosis of pneumatic valve using Adaptive Neuro-Fuzzy Inference System approach, Appl. Soft Comput. J. 19 (2014) 362-371. doi:10.1016/j.asoc.2014.02.008.

[12] M. Kalkat, Experimentally vibration and noise analysis of two types of washing machines with a proposed neural network predictor, Meas. J. Int. Meas. Confed. 47 (2014) 184-192. doi:10.1016/j.measurement.2013.08.039.

[13] G.F. Wang, Y.W. Yang, Y.C. Zhang, Q.L. Xie, Vibration sensor based tool condition monitoring using v support vector machine and locality preserving projection, Sensors Actuators A: Phys. 209 (2014) 24-32. doi:10.1016/j.sna.2014.01.004.

[14] Z. Yin, J. Hou, Recent advances on SVM based fault diagnosis and process monitoring in complicated industrial processes, Neurocomputing. 174 (2016) 643-650. doi:10.1016/j.neucom.2015.09.081.

[15] F. Jia, Y. Lei, J. Lin, X. Zhou, N. Lu, Deep neural networks: A promising tool for fault characteristic mining and intelligent diagnosis of rotating machinery with massive data, Mech. Syst. Signal Process. 72-73 (2016) 303-315. doi:10.1016/j.ymssp.2015.10.025.

[16] K. Worden, W.J. Staszewski, J.J. Hensman, Natural computing for mechanical systems research: A tutorial overview, Mech. Syst. Signal Process. 25 (2011) 4-111. doi:10.1016/j.ymssp.2010.07.013.

[17] B.-S. Yang, W.-W. Hwang, D.-J. Kim, A. Chit Tan, Condition classification of small reciprocating compressor for refrigerators using artificial neural networks and support vector machines, Mech. Syst. Signal Process. 19 (2005) 371-390. doi:10.1016/j.ymssp.2004.06.002.

[18] M. Elhaj, F. Gu, A.D. Ball, A. Albarbar, M. Al-Qattan, A. Naid, Numerical simulation and experimental study of a two-stage reciprocating compressor for condition monitoring, Mech. Syst. Signal Process. 22 (2008) 374-389. doi:10.1016/j.ymssp.2007.08.003.

[19] P. Potočnik, D. Soklič, P. Mužič, M. Absec, T. Strmeč, E. Govekar, Automatic detection of spring faults during assembly of reciprocating compressors, Journal Mech. Eng. 55 (2009) 444-454.

[20] Q. Qin, Z.N. Jiang, K. Feng, W. He, A novel scheme for fault detection of reciprocating compressor valves based on basis pursuit, wave matching and support vector machine, Meas. 45 (2012) 897-908. doi:10.1016/j.measurement.2012.02.005.

[21] Y. Lin, Automated Fault Classification of Reciprocating Compressors from Vibration Data: A Case Study on Optimization using Genetic Algorithm, Procedia Eng. 79 (2014) 355-361. doi:10.1016/j.proeng.2014.06.355.

[22] Y. Wang, C. Xue, X. Jia, X. Peng, Fault diagnosis of reciprocating compressor valve with the method integrating acoustic emission signal and simulated valve motion, Mech. Syst. Signal Process. 56-57 (2015) 197-212. doi:10.1016/j.ymssp.2014.11.002.

[23] K. Pichler, A. Schrems, T. Buchegger, M. Huschenbett, M. Pichler, Fault detection in reciprocating compressor valves under varying load conditions, Mech. Syst. Signal Process. 70-71 (2016) $104-119$. doi:10.1016/j.ymssp.2015.09.005. 
[24] J. Yuan, X. Liu, Semi-supervised learning and condition fusion for fault diagnosis, Mech. Syst. Signal Process. 38 (2013) 615-627. doi:10.1016/j.ymssp.2013.03.008.

[25] Z. Xue, Y. Shang, A. Feng, Semi-supervised outlier detection based on fuzzy rough C-means clustering, Math. Comput. Simul. 80 (2010) 1911-1921. doi:10.1016/j.matcom.2010.02.007.

[26] S. Ghosh, M. Roy, A. Ghosh, Semi-supervised change detection using modified self-organizing feature map neural network, Appl. Soft Comput. J. 15 (2014) 1-20. doi:10.1016/j.asoc.2013.09.010.

[27] X. Wang, H. Feng, Y. Fan, Fault detection and classification for complex processes using semi-supervised learning algorithm, Chemom. Intell. Lab. Syst. 149 (2015) 24-32. doi:10.1016/j.chemolab.2015.10.019.

[28] B. Samanta, Artificial neural networks and support vector machines with genetic algorithm for bearing fault detection, Eng. Appl. Artif. Intel. 16 (2003) 657-665. doi:10.1016/j.engappai.2003.09.006.

[29] V.Ã. Sugumaran, K.I. Ramachandran, Automatic rule learning using decision tree for fuzzy classifier in fault diagnosis of roller bearing, Mech. Syst. Signal Process. 21 (2007) 2237-2247. doi:10.1016/j.ymssp.2006.09.007.

[30] R. Jegadeeshwaran, V. Sugumaran, Fault diagnosis of automobile hydraulic brake system using statistical features and support vector machines, Mech. Syst. Signal Process. 52-53 (2015) $436-446$. doi:10.1016/j.ymssp.2014.08.007.

[31] N. Helwig, S. Klein, A. Schütze, Identification and quantification of hydraulic system faults based on multivariate statistics using spectral vibration features, Procedia Eng. $120 \quad$ (2015) 1225-1228. doi:10.1016/j.proeng.2015.08.835.

[32] I.T. Jolliffe, Principal Component Analysis, second ed., Springer, New York, 2002.

[33] T. Cacoullos, Discriminant Analysis and Applications, Academic Press, New York, 1973.

[34] R.A. Johnson, D.W. Wichern, Applied Multivariate Statistical Analysis, Prentice Hall, New Jersey, 1988.

[35] W.J. Krzanowski, Principles of Multivariate Analysis: A User's Perspective, Oxford University Press, New York, 1988.

[36] G. Dreyfus, Neural Networks, Springer, Berlin Heidelberg, 2005.

[37] K. Chen, L. Wang, eds., Trends in Neural Computation, Springer, Berlin Heidelberg, 2007.

[38] K. Patan, Artificial Neural Networks for the Modelling and Fault Diagnosis of Technical Processes, Springer, Berlin Heidelberg, 2008.

[39] S. Haykin, Neural Networks and Learning Machines, third ed., Pearson Education, Upper Saddle River, New Jersey, 2009.

[40] M.T. Hagan, M.B. Menhaj, Training feed forward networks with the Marquardt algorithm, IEEE Trans. Neural Networks. 5 (1994) 989-993.

[41] D.J.C. MacKay, Bayesian interpolation, Neural Comput. 4 (1992) 415-447.

[42] J. Moody, C.J. Darken, Fast learning in networks of locally-tuned processing units, Neural Comput. 1 (1989) 281294.

[43] C.J.C. Burges, A Tutorial on Support Vector Machines for Pattern Recognition, Data Mining and Knowledge Discovery. 2 (1998) 121-167.

[44] V.N. Vapnik, The Nature of Statistical Learning Theory, Springer, New York, 1995.

[45] V.N. Vapnik, Statistical Learning Theory, John Wiley \& Sons, 1998.

[46] C. Cortes, V. Vapnik, Support-Vector Networks, Mach. Learn. 20 (1995) 273-297. doi:10.1023/A:1022627411411.

[47] G.-B. Huang, Q.-Y. Zhu, C.-K. Siew, Extreme learning machine: Theory and applications, Neurocomputing. 70 (2006) 489-501. doi:10.1016/j.neucom.2005.12.126.

[48] S. Ding, X. Xu, R. Nie, Extreme learning machine and its applications, Neural Comput. Appl. 25 (2014) 549-556. doi:10.1007/s00521-013-1522-8.

[49] G. Huang, G.-B. Huang, S. Song, K. You, Trends in extreme learning machines: A review, Neural Networks. 61 (2015) 32-48. doi:10.1016/j.neunet.2014.10.001.

[50] G.-B. Huang, L. Chen, Convex incremental extreme learning machine, Neurocomputing. 70 (2007) 3056-3062. doi:10.1016/j.neucom.2007.02.009.

[51] G.-B. Huang, L. Chen, C.K. Siew, Universal approximation using incremental constructive feedforward networks with random hidden nodes, IEEE Trans. Neural Networks. 17 (2006) 879-892. doi:10.1109/TNN.2006.875977.

[52] X. Liu, C. Gao, P. Li, A comparative analysis of support vector machines and extreme learning machines, Neural Networks. 33 (2012) 58-66. doi:10.1016/j.neunet.2012.04.002.

[53] P. Potočnik, E. Govekar, Practical Considerations in Training Extreme Learning Machines, in: 16th Int. Conf. Eng. Appl. Neural Networks, Rhodes Island, Greece, 2015. 